\title{
OS SINTOMAS DEPRESSIVOS E A FRAGILIDADE FÍSICA EM IDOSOS
}

Aline de Sousa Falcão; Universidade Federal do Paraná (UFPR); E-mail: alinesousafalcão@hotmail.com;

Conceição da Silva Brito; UFPR; E-mail: enfbryto@gmail.com;

Márcia Marrocos Aristides Barbiero; UFPR; E-mail: marciamarrocos@gmail.com;

Patrícia Rosa Gonçalves Leta; UFPR; E-mail: patriciarg|@hotmail.com;

Reuber Lima de Sousa; UFPR; E-mail: reuber_souza@hotmail.com;

Karina Silveira de Almeida Hammerschmidt; UFPR; E-mail: ksalmeidah@ufpr.br; Susanne Elero Betiolli; Universidade Federal do Paraná; susanne.elero@yahoo.com.br;

Maria Helena Lenardt; UFPR; E-mail: curitiba.helena@gmail.com

\section{RESUMO}

Introdução: A fragilidade física na pessoa idosa pode levar ao desenvolvimento de condições clínicas críticas e agravos à saúde, entre eles o surgimento de sintomas depressivos. O conhecimento cientifico a respeito do comportamento e da relação entre essas variáveis ainda é pouco esclarecido. Objetivo: identificar a relação entre sintomas depressivos e fragilidade física em idosos. Métodos: trata-se de uma revisão integrativa da literatura desenvolvida a partir de 24 artigos selecionados mediante critérios de inclusão e exclusão pré-estabelecidos, que foram considerados por estar publicado no período de 2012 a 2020, constar na íntegra, estar diretamente relacionados ao tema, e estar registrado nos idiomas português e/ou inglês. Para a seleção dos artigos utilizou-se o Preferred Reporting Items for Systematic Reviews and Metaanalyses, assim como o Oxford Centre for Evidence-based Medicine para a classificação do nível de evidência e o EndNote Web para gerenciamento da bibliografia. As busca dos artigos ocorreu em 4 bases de dados: PUBMED, Web of Science, BVS e SciELO. Resultados: observou-se associação entre os sintomas depressivos e a fragilidade física em idosos; A fragilidade física foi considerada um preditor de sintomas depressivos; Os indivíduos frágeis podem ser mais propensos a desenvolver sintomas depressivos devido às habilidades funcionais prejudicadas, inatividade física, incapacidade funcional e isolamento social. Conclusão: os resultados auxiliam no esclarecimento da relação entre os sintomas depressivos e a fragilidade física, ao tempo em que gera um alerta para a importância da identificação da fragilidade física na prática clínica, como rastreamento de idosos com risco para sintomas depressivos.

Palavras-chave: Idoso Fragilizado; Depressão; Saúde do Idoso. 